\title{
Anabases
}

ANABASES Traditions et réceptions de l'Antiquité

4 | 2006

Varia

\section{Le musée Saint-Raymond, musée des Antiques de Toulouse, et ses portraits romains}

Adeline Grand-Clément

\section{(2) OpenEdition}

1 Journals

Édition électronique

URL : http://journals.openedition.org/anabases/3023

DOI : 10.4000/anabases.3023

ISSN : 2256-9421

Éditeur

E.R.A.S.M.E.

Édition imprimée

Date de publication : 1 octobre 2006

Pagination : 280-287

ISSN : 1774-4296

\section{Référence électronique}

Adeline Grand-Clément, « Le musée Saint-Raymond, musée des Antiques de Toulouse, et ses portraits romains », Anabases [En ligne], 4 | 2006, mis en ligne le 01 janvier 2012, consulté le 20 octobre 2019. URL : http://journals.openedition.org/anabases/3023 ; DOI : 10.4000/anabases.3023

Ce document a été généré automatiquement le 20 octobre 2019.

(c) Anabases 


\title{
Le musée Saint-Raymond, musée des Antiques de Toulouse, et ses portraits romains
}

\author{
Adeline Grand-Clément
}

\section{En guise de présentation}

1 L'histoire de la réception de l'Antiquité se nourrit de celle de l'accueil réservé aux œuvres antiques, qui contribuent tout autant que les textes à la connaissance des civilisations passées. Au cours du XIXe siècle, la «naissance » de l'archéologie moderne modifie profondément le regard porté sur les objets antiques, amorçant une évolution du statut qui leur est dévolu. La recherche du «bel objet ", œuvre d'art élevée au rang de modèle indépassable, qui suscite admiration et invite à la contemplation, laisse la place à celle de l'artefact, comme témoignage de la culture matérielle d'une société donnée.

2 En Europe occidentale, l'évolution s'accompagne d'une floraison de musées, qui remplacent les cabinets de curiosités et ont pour fonction d'abriter les collections d'antiques, en les rendant accessibles à un public de plus en plus élargi. Le souci initial de conservation et de valorisation du patrimoine se double rapidement d'une volonté de doter ces établissements d'une fonction pédagogique, qui s'est accrue au fil du temps. Il ne s'agit plus seulement de montrer, de donner à voir, mais aussi de permettre de comprendre : sortir le visiteur de sa passivité, afin que s'instaure entre lui et l'objet un dialogue fécond. Enfin, la mission scientifique dévolue aux musées leur impose de prendre en compte et de diffuser auprès du public l'actualité de la recherche scientifique: les établissements sont des lieux ouverts, vivants, qui apportent leur contribution à l'atelier de l'Histoire.

3 Depuis le XIXe siècle, les choix effectués par les conservateurs au sein des collections de leurs musées, pour sélectionner les objets susceptibles d'être présentés, ne sont pas neutres et contribuent à élaborer et à diffuser une certaine image de l'Antiquité au sein 
du public. La muséographie et la rédaction des cartels - ces courtes notices destinées à rendre les objets intelligibles - jouent également un rôle de médiation déterminant entre l'objet et le visiteur.

4 L'objectif de cette rubrique consistera donc à mettre en lumière les diverses stratégies adoptées dans les musées européens pour « donner à voir » l'Antiquité, en liaison avec les avancées de la recherche scientifique et les progrès des découvertes archéologiques. Partant à chaque fois à la rencontre d'une personne ou d'une équipe ayant en charge un établissement, nous nous attacherons à présenter les ressources d'un fonds spécifique et les initiatives originales adoptées afin de le présenter au public.

5 Nous avons choisi, pour inaugurer la rubrique, de porter notre attention sur le musée Saint-Raymond, musée des Antiques de Toulouse, qui abrite l'une des quatre plus importantes collections d'antiquités de France. Il a présenté en 2005-2006 l'exposition Portraits $d u I^{e r}$ siècle de l'empire romain, qui inaugure un cycle consacré aux portraits romains.

\section{Le musée Saint-Raymond, musée des Antiques de Toulouse, et ses portraits romains}

Lors de l'inauguration du musée, en 1892, le maire de Toulouse, Camille Ournac, affiche un programme ambitieux, à la mesure de l'idéal républicain :

7 La création de ce musée est une œuvre essentiellement démocratique dans le sens le plus élevé du mot; et c'est utilement, à mon avis, travailler pour le peuple, que de lui enseigner l'histoire par les yeux : c'est former son goût, que de lui inculquer l'amour du beau sous toutes ses formes, toutes choses qui rendent l'homme meilleur ${ }^{1}$.

8 Les rencontres avec Daniel Cazes, le conservateur en chef, et Aurélie Rodes, chargée de mission au Service éducatif du musée, confirment que le défi a été relevé ${ }^{2}$. Depuis sa réouverture en 1999, le musée accueille plus de cinquante mille visiteurs par an.

\section{Rencontre avec Daniel Cazes, conservateur en chef}

9 - Le musée des Antiques de Toulouse et ses collections : bref aperçu historique

L'histoire du musée et de la constitution de ses collections a fait l'objet de plusieurs publications, dirigées par Daniel Cazes ${ }^{3}$. Elle commence en 1795, avec l'ouverture au public du Museum provisoire du Midi de la République, installé dans l'ancien couvent des Augustins. Dès le début du XIX ${ }^{\mathrm{e}}$ siècle, on isole, au sein de l'ensemble des collections d'œuvres d'art, une collection d'« antiquités ", qui fait l'objet d'une présentation spécifique dans les galeries du cloître. L'initiative conduit finalement à la création, sous la houlette d'Alexandre Du Mège, d'un département indépendant de celui des beauxarts, qui reçoit alors le nom de « Musée des Antiques».

À la fin du XIX $X^{e}$ siècle, le musée des Augustins se trouve dans l'incapacité matérielle d'abriter et de présenter l'ensemble des collections municipales. La mairie choisit alors de transférer une partie de celles-ci, en particulier les "petites antiquités", rassemblant divers objets égyptiens, grecs et romains, dans un ancien collège universitaire du XIII ${ }^{\mathrm{e}}$ siècle, le collège Saint-Raymond, situé à côté de la basilique SaintSernin. En 1892, le maire de Toulouse inaugure le musée, qui conserve alors le nom de l'établissement. C'est au cours des années 1940, lorsque la mairie procède à une 
réorganisation générale des collections au sein des différents musées, sur la base de critères chronologiques, que l'établissement se spécialise dans l'Antiquité classique et le haut Moyen Âge ${ }^{4}$.

11 Rebaptisé récemment "musée des Antiques de Toulouse », dans un souci d'assurer une meilleure visibilité auprès du public, tout en renouant avec son histoire, l'établissement dispose d'un fonds très riche, constitué depuis la Révolution française sur la base des saisies révolutionnaires. Il a été alimenté au fil du temps par les ventes et les dons des érudits collectionneurs et des sociétés savantes, notamment la Société Archéologique du Midi de la France, fondée en 1831. Le musée a également bénéficié du résultat des campagnes de fouilles archéologiques, certaines anciennes, en particulier à Chiragan, au sud-ouest de Toulouse, d'autres très récentes, comme celles qui ont été menées dans la ville à l'occasion des travaux d'urbanisme, dans les années 1990.

En dépit des travaux de rénovation et d'agrandissement effectués récemment, l'exiguïté de l'espace d'exposition ne permet pas de présenter au public les dizaines de milliers d'objets conservés. Lors de la réouverture du musée, en 1999, Daniel Cazes a donc été confronté à un choix. Il ne s'agissait plus, comme jusque dans les années 1950, de chercher à tout montrer, en entassant les objets. Partant du principe que le visiteur se trouve à Toulouse et désire sans doute approfondir sa connaissance du lieu, le conservateur a jugé judicieux de privilégier la dimension locale, afin de d'esquisser un parcours cohérent et dialectique. Une telle décision offrait aussi l'avantage de justifier l'intégration des données propres au site, en particulier les découvertes archéologiques de la nécropole funéraire associée à la basilique Saint-Sernin, toute proche. Le public découvre donc au fil des étages l'histoire de la ville antique de Tolosa et de son territoire, jusqu'à l'époque paléochrétienne, ainsi que les éléments contextuels nécessaires à la compréhension, en particulier son intégration au sein de la province de Narbonnaise. De fait, les nombreux objets de provenance inconnue ou issus d'autres régions de l'Empire romain, la très riche série de monnaies, ou encore l'importante collection d'œuvres grecques, en particulier les vases du fonds Campana, ne sont pas présentés de façon permanente. Cela ne signifie pas pour autant qu'ils sont inaccessibles. Ils peuvent être intégrés à l'occasion d'expositions temporaires. De plus, toute personne intéressée dispose d'un droit d'accès aux réserves : Daniel Cazes a confié à l'un des membres de son équipe, Claudine Jacquet, assistante de conservation du patrimoine, la charge d'accueillir ceux qui en feraient la demande.

13 - Les missions du musée et l'attention portée à l'accueil du public

Aujourd'hui, les missions confiées aux musées associent étroitement trois aspects complémentaires : la conservation, la présentation et l'étude des objets dont ils ont la charge. Le premier volet vise à assurer leur transmission aux générations futures. Les professionnels chargés de la restauration interviennent donc lorsque l'état d'un objet l'exige. Il ne s'agit plus, comme en avaient pris l'habitude les antiquaires du $\mathrm{XIX}^{\mathrm{e}}$ siècle, de vouloir reconstituer à tout prix des œuvres entières, afin d'exposer de «beaux objets»- combien de portraits antiques ont ainsi été affublés d'un nez moderne! Seul le document authentique, tel qu'il nous est parvenu, compte. Daniel Cazes souligne que la nécessité de préserver le patrimoine public est une question de déontologie pour les conservateurs de monuments, de sites et de musées. Selon lui, une telle préoccupation doit également primer lors des fouilles archéologiques. Les relevés, les photographies, les ressources de l'informatique ne peuvent remplacer l'objet original, le seul objet qui intéresse véritablement l'historien. 
14 Le deuxième aspect, celui de la présentation, soulève un certain nombre de questions cruciales : comment exposer? comment expliquer ? à qui s'adresser ? Daniel Cazes estime que l'on a trop souvent tendance à négliger les implications sociales des musées : il s'agit de lieux de rencontre privilégiés, où le visiteur vient chercher des réponses à ses propres interrogations personnelles. Le conservateur ne peut ignorer cette réalité lorsqu'il réfléchit à l'aménagement de son musée et à la présentation des collections. Lorsqu'il procède à des choix muséographiques, il doit aussi tenir compte de l'histoire de l'établissement, de son implantation géographique, de la nature de ses collections - en un mot, de tout ce qui lui confère sa "personnalité ». Daniel Cazes déplore les tendances actuelles de la muséographie, qui conduisent à uniformiser les modes de présentation, en ignorant les spécificités propres à chaque site.

15 Depuis son arrivée, il veille à garantir à tous, au-delà des différences d'âge, de profession ou de nationalité, l'accès aux collections publiques dont il a la charge. Il estime que le rôle du musée est de s'adresser à l'ensemble de la collectivité, mais aussi de lui rendre compte très vite des progrès de la recherche scientifique, menée à bien grâce au financement public. Dans cette perspective, les choix muséographiques doivent être constamment réévalués et adaptés, dans un souci de pédagogie et d'actualisation permanente. Aussi le programme élaboré en 1995-1996, pour la réouverture en 1999, va-t-il bientôt faire l'objet d'une révision, qui intègrera les avancées de la recherche menée au sein des universités et du CNRS, mais aussi à l'intérieur du musée lui-même, puisque l'étude scientifique s'inscrit dans le cadre du troisième volet de sa sphère d'activité.

16 Selon les propres mots de Daniel Cazes, « un musée qui ne fait pas de recherche au sein de sa propre équipe est un musée mort ». L'équipe scientifique, qui consacre une partie de son travail à l'étude des objets, rend ses résultats publics de deux façons : par des expositions; par les publications qui les accompagnent. Daniel Cazes insiste sur la quantité de travail qui précède la mise en place d'une exposition: rassembler la documentation scientifique sur le sujet, la synthétiser et la convertir en un langage accessible au plus grand nombre, afin d'élaborer des cartels clairs et utiles. Le musée a également pour mission la publication de catalogues raisonnés, à l'attention des chercheurs, français et étrangers, afin de mettre à leur disposition les informations nécessaires sur les collections.

- La conception et la mise en place de l'exposition Portraits du $\mathrm{i}^{\mathrm{er}}$ siècle de l'empire romain

Dans les collections du musée, les portraits romains occupent une place de choix: l'établissement abrite en effet la plus importante collection de portraits romains découverts en France. La majeure partie sont d'une qualité exceptionnelle, en particulier deux ensembles : celui mis au jour au cours du XIX siècle dans la villa de Chiragan, à Martres Tolosane (Haute-Garonne), qui rassemble des œuvres datant du $\mathrm{I}^{\mathrm{er}}$ siècle avant J.-C. au $\mathrm{V}^{\mathrm{e}}$ siècle après J.-C., et celui provenant du forum de Béziers, qui représente les membres de la domus augustea. Tandis que le second a fait l'objet d'une publication récente ${ }^{5}$, les études consacrées au premier méritaient d'être actualisées : les ouvrages de références sont aujourd'hui anciens et dépassés ${ }^{6}$. Daniel Cazes a perçu la nécessité d'offrir à la communauté scientifique un nouveau catalogue raisonné des sculptures de Chiragan. Mettant ses compétences au service de l'entreprise, il s'est assuré la collaboration de Jean-Charles Balty, spécialiste de la sculpture antique, notamment du portrait. Le projet initial de produire un catalogue rassemblant de courtes notices a rapidement été abandonné, lorsque Daniel Cazes a pris conscience que 
la collection était universellement connue et qu'il s'avérait nécessaire de fournir une bibliographie scientifique complète, donc de reprendre la totalité du dossier, complexe et délicat, des fouilles menées depuis le XIX ${ }^{e}$ siècle. Le volume paru en 2005 concerne uniquement l'époque julio-claudienne ${ }^{7}$ et constitue le premier volet d'une série intitulée Sculptures antiques de Chiragan Les portraits romains, qui en comportera six, afin de couvrir un champ chronologique large, jusqu'à l'époque théodosienne. Il présente les résultats fructueux de vingt ans de recherche, avec des hypothèses nouvelles et quelques découvertes majeures, telle l'identification du portrait de Tiberius Gemellus.

Daniel Cazes ne concevait pas de limiter l'initiative au projet scientifique. Il a souhaité accompagner la publication du catalogue d'une exposition temporaire, afin de tenir le public informé des recherches menées dans le domaine. Elle intègre toutes les œuvres cataloguées, en y ajoutant divers objets qui permettent de les replacer dans leur contexte historique et d'établir des parallèles utiles: en particulier une série de monnaies émises par les Julio-Claudiens et d'autres éléments de sculpture contemporains. Bien qu'un soin particulier ait été apporté à la mise en valeur esthétique des objets, le conservateur n'a pas souhaité que l'exposition prenne la forme d'une leçon d'histoire de l'art: elle aborde des questions d'histoire politique et sociale. L'objectif est de sensibiliser le public aux procédés employés par la propagande augustéenne et à la façon dont elle a été reçue localement, c'est-à-dire à Tolosa et en Narbonnaise. L'exposition a notamment permis de sortir des réserves divers portraits, fabriqués dans des ateliers locaux du sud de la Gaule, afin de montrer qu'ils s'inspiraient des modèles officiels élaborés à Rome.

19 L'exposition a fermé ses portes en mai 2006. Durant la dernière semaine, elle a abrité, aux côtés des originaux, des portraits réalisés par des scolaires, qui ont participé à un concours de caricatures organisé par le service éducatif du musée. Les productions des élèves ont apporté un souffle de couleurs et de dynamisme aux cuvres antiques. Cette initiative originale, qui affirme avec force que le musée demeure un lieu ouvert, a permis d'une certaine façon de rappeler que les sculptures, autrefois rehaussées de polychromie, occupaient une place vivante dans le quotidien des Romains.

\section{Le service éducatif du musée : questions à Aurélie Rodes, professeur agrégée, chargée de mission au Service éducatif du musée pour l'Action culturelle du Rectorat}

\section{- Qu'est ce qu'un service éducatif?}

Le terme « service éducatif » recouvre en fait des situations diverses. Nés d'initiatives isolées, « l'action éducative » ou « le service pédagogique » ont été intégrés aux services des publics, rendus obligatoires par la loi du 4 janvier 2002 relative aux musées de France. Les actions menées en direction du jeune public sont confiées à des professionnels du patrimoine et de la conservation, des médiateurs culturels, et plus rarement des enseignants du secondaire, chargés de mission par l'Éducation nationale pour organiser l'accueil des groupes scolaires ${ }^{8}$. Ils font le lien entre deux univers professionnels. Il s'agit de faire connaître le milieu du musée et de l'archéologie aux enseignants et faire comprendre les contraintes spécifiques du monde de l'éducation aux conservateurs.

21 Longtemps, les musées n'ont proposé aux visiteurs, y compris aux plus jeunes, que des visites guidées, assurées par des conférenciers. Un service éducatif animé par un 
enseignant a pour objectif de proposer une alternative à ce type de visite, en aidant les professeurs à préparer et à organiser des visites autonomes et gratuites au musée avec leurs classes. Son action intervient donc avant, pendant et après la venue des groupes. Dans un premier temps, des rencontres avec les enseignants ont lieu de façon régulière, afin de leur permettre de se familiariser avec les collections du musée, les monuments, les sites et les expositions temporaires. Une documentation succincte, des mallettes pédagogiques et des propositions d'activités plus ou moins ludiques - jeu, enquêtes, parcours découverte - sont ensuite mises à leur disposition. En dehors des visites ellesmêmes, des ateliers, conçus par le Service éducatif et animés par des professionnels, en lien avec les collections permanentes, permettent aux élèves de découvrir la matérialité des objets : sculpture, mosaïque, céramique, monnaie...

La présence d'un service éducatif dans un musée permet de faire évoluer les pratiques des enseignants au musée. Les élèves sont de plus en plus autonomes : en renonçant à tout leur montrer, on leur permet de s'attarder sur quelques œuvres qui font sens. La visite n'est plus considérée comme une sortie récréative de fin d'année, mais comme un travail sur des documents originaux, qui a fait l'objet d'une préparation, en amont, et peut ensuite être réexploitée en classe. Le service éducatif peut aussi intervenir dans la muséographie. Lors de l'exposition Gaulois des pays de Garonne, présentée de mai 2004 à janvier 2005, les cartels, bien que rédigés pour un large public, restaient complexes pour de jeunes élèves. Des ateliers, encadrés par une assistante de conservation du patrimoine, leur ont donné l'occasion de relever tout ce qu'ils ne comprenaient pas dans l'exposition. Après un travail de recherche, ils ont rédigé des notices explicitant les points qui leur avaient paru obscurs. Leurs productions écrites et graphiques ont ensuite été intégrées à l'exposition, pour proposer au visiteur un autre niveau de lecture.

Nous avons souhaité prolonger l'expérience au-delà du musée et promouvoir toutes les initiatives innovantes d'enseignants destinées à faire aimer l'Antiquité à leurs élèves. Le 31 mai 2006, pour la première fois en Midi-Pyrénées, s'est tenu à l'université de Toulouse Le Mirail un "forum antique », qui a permis à différentes classes d'échanger sur les projets auxquels ils ont participé au cours de l'année scolaire, en liaison avec le musée. Les élèves du collège Maurice Bécanne ont ainsi présenté le sixième numéro de leur "Journal de l'olympe", que le musée met gratuitement à la disposition des visiteurs.

24 - Comment avez-vous accompagné l'exposition Portraits du $\mathrm{I}^{\mathrm{er}}$ siècle de l'empire romain?

Les expositions temporaires du musée ne sont pas conçues avec la seule finalité d'attirer un public scolaire. Les conservateurs ont pour mission de présenter les collections à un large public, mais aussi de faire partager des connaissances scientifiques à un public plus spécialisé ${ }^{9}$. Afin de permettre au public scolaire d'avoir accès à des expositions parfois difficiles, différents dispositifs sont proposés. Pour cette exposition, les élèves devaient identifier des portraits à partir d'un corpus documentaire simple et mettre en relation les profils des monnaies et les portraits sculptés. Pour la première fois, il leur a été proposé de s'intéresser aux œuvres, par le biais d'un concours de caricature. L'idée est née lors d'une séance de séminaire de l'équipe Érasme au musée, sur le thème "Enseigner l'Antiquité des Anciens aux modernes ». À cette occasion, nous avions engagé une discussion sur les enjeux politiques d'une muséographie qui ne serait pas affranchie du discours impérial ${ }^{10}$. Cela m'a amenée à m'interroger sur l'impact que pouvaient encore avoir ces œuvres de 
propagande sur de jeunes visiteurs. La caricature apparaissait comme le moyen de s'approprier les œuvres et de prendre conscience de la nature de l'idéologie impériale. La qualité des créations et l'implication de chacun ont suscité l'enthousiasme du jury, composé de professionnels de la culture et de l'éducation. Pour la première fois, grâce à l'audace de Daniel Cazes, les productions d'élèves ont été présentées au sein du musée, à côté des originaux.

Pour tout contact : Musée Saint-Raymond, musée des Antiques de Toulouse Place Saint-Sernin 31000 Toulouse

Tél. 0561223144

Service éducatif : http://pedagogie.ac-toulouse.fr/culture/saintraymond/ straymenu.htm

\section{NOTES}

1. D’après D. CAZES, Le Musée Saint-Raymond: 1892-1992, Toulouse, Musée Saint-Raymond, 1992, p. 21.

2. Je tiens à leur exprimer de chaleureux remerciements pour l'excellent accueil qu'ils ont bien voulu me réserver.

3. D. Cazes a mené un patient et minutieux travail d'archéologie des collections, en particulier celles de sculpture : parmi les principales références, citons D. CAZES, Le Musée Saint-Raymond; D. CAZES et alii, Le Musée Saint-Raymond, musée des Antiques de Toulouse, Toulouse-Paris, Musée SaintRaymond-Somogy, 1999.

4. Les sarcophages paléochrétiens, longtemps conservés au musée des Augustins, n'ont rejoint le musée Saint-Raymond qu'après 1990.

5. J.-C. BALTY et D. CAZES, Portraits impériaux de Béziers. Le groupe statuaire du forum, Toulouse, Musée Saint-Raymond, 1995.

6. La principale référence bibliographique utilisée jusque là par les historiens de l'art français et étrangers remonte au début $\mathrm{du} x \mathrm{xx}^{\mathrm{e}}$ siècle : L. Joulin, "Les établissements gallo-romains de la plaine de Martres Tolosane", Mémoires présentés par divers savants à l'Académie des Inscriptions et Belles Lettres 11 (1901), p. 219-516.

7. J.-C. BALTY et D. CAZES, Sculptures antiques de Chiragan, I : Les portraits romains, I, 1 : Époque julioclaudienne, Toulouse, Musée Saint-Raymond, 2005.

8. L'Académie de Toulouse est bien dotée et le musée des Antiques dispose, depuis sa réouverture en mai 1999, d'un demi-poste d'enseignant, pour les collections permanentes, les expositions temporaires, les monuments et les sites (l'ancienne église Saint-Pierre-des-Cuisines, la basilique Saint-Sernin, l'amphithéâtre romain de Purpan et la natatio des thermes d'Ancely).

9. Ainsi, l'exposition Gaulois des pays de Garonne a été inaugurée au moment où s'est tenu, à Toulouse, un colloque sur l'Âge du fer. De même, l'exposition Portraits du I Ir siècle de l'empire romain s'inscrit dans le cadre d'une publication scientifique de l'étude des collections.

10. Les portraits d'empereurs présentés au premier étage ont été rassemblés en enfilade, formant ainsi une galerie qui encadre et impressionne le visiteur lors de son parcours. 


\section{AUTEUR}

\section{ADELINE GRAND-CLÉMENT}

Université de Toulouse II - Le Mirail

adddeline@hotmail.com 